\title{
RENDIMIENTO Y ADAPTACIÓN DE LA VARIEDAD DE FRIJOL 'NEGRO COMAPA' EN DOS REGIONES DE MÉXICO
}

\author{
SEED YIELD AND ADAPTABILITY OF 'NEGRO COMAPA' BEAN CULTIVAR IN \\ TWO MÉXICAN REGIONS
}

\author{
Ernesto López Salinas ${ }^{1}$, Óscar H. Tosquy Valle', Yanet Jiménez Hernández², Rafael A. Salinas Pérez ${ }^{3}$, \\ Bernardo Villar Sánchez ${ }^{4}$ Jorge A. Acosta Gallegos ${ }^{2 *}$
}

\begin{abstract}
${ }^{1}$ Campo Experimental Cotaxtla, Instituto Nacional de Investigaciones Forestales, Agrícolas y Pecuarias (INIFAP). Km 34.5 Carr. Federal Veracruz-Córdoba, Apartado Postal 429. 91700, Veracruz, Ver. ${ }^{2}$ Campo Experimental Bajío, INIFAP. Km 6.5 Carr. Celaya-San Miguel de Allende. 38110, Celaya, Gto. ${ }^{3}$ Campo Experimental Valle del Fuerte, INIFAP. km 1609 Carr. Internacional, Juan José Ríos, Sin. ${ }^{4}$ Campo Experimental Centro de Chiapas, INIFAP. Km 3.0 Carr. OcozocoautlaCintalapa. 29140, Ocozocoautla, Chis.
\end{abstract}

${ }^{*}$ Autor para correspondencia (acosta.jorge@inifap.gob.mx)

\section{RESUMEN}

El frijol negro (Phaseolus vulgaris L.), de grano opaco y pequeño es de alta demanda en las regiones del centro y sureste de México. El objetivo de esta investigación fue determinar la adaptación y el rendimiento de la nueva variedad de frijol 'Negro Comapa' en áreas del trópico húmedo del Golfo y en el altiplano central de México. De 2007 a 2010 se estableció un ensayo de rendimiento de líneas de frijol que incluyó a esta variedad en seis ambientes del Estado de Veracruz (dos bajo temporal o secano, y cuatro bajo condiciones de humedad residual). En 2009 y 2010 también se evaluó en un ensayo nacional de rendimiento, en 13 ambientes de prueba en México: seis en Veracruz, cuatro en Guanajuato, uno en Durango uno en Sinaloa y uno en Chiapas. En el ensayo nacional, además del rendimiento se determinó la reacción de las variedades a las enfermedades que incidieron en forma natural. En los años mencionados, 'Negro Comapa' también se evaluó en pruebas de validación en terrenos de agricultores en 10 ambientes de Veracruz, uno en Oaxaca y uno en Chiapas. En ambos ensayos, el conducido en Veracruz y el nacional, 'Negro Comapa' mostró un rendimiento promedio de 1287 y $1764 \mathrm{~kg} \mathrm{ha}^{-1}$, respectivamente, que superó al de los demás genotipos. En los sitios con presión por enfermedades, 'Negro Comapa' resultó resistente al virus mosaico común (BCMV) y a la mancha angular, y tolerante al virus del mosaico amarillo dorado del frijol (BGYMV). En parcelas de validación, el rendimiento promedio de 'Negro Comapa' fue $51 \%$ mayor que el testigo del agricultor.

Palabras clave: Phaseolus vulgaris, frijol negro, rendimiento, adaptabilidad, tolerancia a enfermedades foliares.

\section{SUMMARY}

Black beans (Phaseolus vulgaris L.), with opaque and small grains, are in high demand in the regions of Central and Southeastern regions of México. The present research had the objective of determining the adaptation and yield of the new bean variety 'Negro Comapa' in the humid tropical areas of the Gulf of México and in the Central Mexican highlands. From 2007 to 2010, a regional trial that included this variety was established in six environments in the state of Veracruz (two under rain-fed conditions and four under residual moisture conditions). In 2009 and 2010 this variety was also included in a national yield trial which was evaluated in 13 test environments in México: six in Veracruz, four in Guanajuato, one in Durango, one in Sinaloa, and one in Chiapas. In the national test, in addition to yield, the response of the variety to naturally incident diseases was determined. In the same years 'Negro Comapa' was validated in farmer fields in 10 environments of Veracruz, one in Oaxaca and one in Chiapas. In both trials, the one conducted in Veracruz and the national one, 'Negro Comapa' showed higher average yield, outperforming all other genotypes, with a yield of 1287 and 1764 $\mathrm{kg} \mathrm{ha}^{-1}$, respectively. At sites with disease pressure, 'Negro Comapa' was resistant to common mosaic virus (BCMV) and angular leaf spot, and tolerant to the bean golden yellow mosaic virus (BGYMV). In validation plots, the average yield of 'Negro Comapa' was $51 \%$ higher than the famers' variety.

Index words: Phaseolus vulgaris, yield, adaptability, tolerance to foliar diseases.

\section{INTRODUCCIÓN}

El frijol negro (Phaseolus vulgaris L.), tanto el brillante de tamaño medio como el pequeño y opaco, es el tipo de frijol de mayor demanda en México; su mercado es muy amplio en el centro y en el sureste del país, que asciende a 400000 toneladas anuales (Castellanos et al., 1997; SIAP, 2006). Actualmente los principales estados productores de de frijol negro en la región tropical húmeda son Chiapas y Veracruz, en la región del trópico seco son Nayarit y Sinaloa, y en el altiplano semiárido son Zacatecas, Durango y Guanajuato (Acosta et al., 2000; López et al., 2011). En los dos primeros estados se siembra frijol en condiciones de temporal (secano) y de humedad residual; en Nayarit principalmente en esta última condición, mientras que en los estados del altiplano predominan las siembras de temporal (SAGARPA, 2011).

Como el frijol de grano negro opaco de raza Mesoamericana (Singh et al., 1991) es neutral al fotoperiodo, la mayoría de variedades mejoradas existentes pueden sembrarse en cualquier época del año en las diferentes zonas agroecológicas del país. Sin embargo, en la gran mayoría de las siembras comerciales aún se utilizan genotipos criollos de adaptación específica y materiales importados como la variedad 'Negro Michigan' que es susceptible a deficiencias 
de humedad y a enfermedades. Lo anterior repercute en bajos rendimientos, y por ende en altos costos por tonelada producida (López et al., 2006; Acosta et al., 2008; Villar y López, 2009).

Para contribuir a solucionar esta problemática, se han desarrollado nuevas variedades mejoradas, con características agronómicas deseables, con rendimiento y calidad superiores al de las variedades comerciales actuales; así se generó la variedad 'Negro Comapa', la cual ha mostrado alta productividad, tolerancia a enfermedades y amplia adaptación en las diferentes áreas en donde se produce frijol en Veracruz y otros estados del país.

La variedad 'Negro Comapa' se originó de la cruza de tres líneas: (VAX-4/A-801)//DOR-500, realizada en el Centro Internacional de Agricultura Tropical (CIAT) en Cali, Colombia. La línea VAX-4 se utilizó como fuente de resistencia a bacteriosis común (X. axonopodis pv. phaseoli) (Singh y Schwartz, 2010). La línea A-801 es resistente a la mancha angular (Phaeoisariopsis griseola), y DOR-500 al virus del mosaico amarillo dorado del frijol (BGYMV) y roya (Uromyces appendiculatus var. appendiculatus) (López et al., 2010).

La familia de la cual se derivó 'Negro Comapa' se introdujo a México en 2002 con el código MN13337-9, a través de un proyecto colaborativo. De 2003 a 2005 se evaluó en viveros de adaptación establecidos en los Estados de Veracruz y Chiapas con el código CIAT-103-21, y fue seleccionada por su alto rendimiento en ensayos uniformes de rendimiento y validación semi-comercial. El objetivo de esta investigación fue determinar la adaptación, rendimiento y reacción a enfermedades de la variedad 'Negro Comapa' en áreas del trópico húmedo y en el altiplano de México.

\section{MATERIALES Y MÉTODOS}

\section{Rendimiento y adaptación de la variedad 'Negro Comapa'}

De 2007 a 2010, 'Negro Comapa' (CIAT-103-21) se evaluó en un ensayo de rendimiento regional (ERR) conformado por 16 genotipos de frijol negro, el cual se estableció en seis sitios en el Estado de Veracruz: dos en condiciones de temporal (uno en Córdoba y otro en Comapa) y cuatro con humedad residual (dos en Medellín de Bravo, uno en Juan Rodríguez Clara y uno en Ixtacuaco). El ensayo incluyó ocho líneas desarrolladas por el programa de mejoramiento del Instituto Nacional de Investigaciones Forestales, Agrícolas y Pecuarias (INIFAP) y seis del programa de frijol del CIAT, la variedad comercial 'Negro Jamapa' y un criollo regional, bajo un diseño experimental de bloques completos al azar con tres repeticiones.
La unidad experimental fue de tres surcos de $5 \mathrm{~m}$ de longitud, con una separación de $0.60 \mathrm{~m}$, y la parcela útil fue el surco central completo. El manejo agronómico del ensayo regional se efectuó con base en las recomendaciones del Campo Experimental Cotaxtla del INIFAP (López et al., 1994). El grano cosechado se limpió, pesó, determinó su humedad y se estimó el rendimiento de grano en kilogramos por hectárea a $14 \%$ de humedad. El rendimiento de los genotipos en los seis experimentos se analizó en forma combinada con el paquete estadístico de la Universidad Autónoma de Nuevo León (Olivares, 1994), y para la comparación del rendimiento de cada tratamiento individual contra el testigo se utilizó la prueba de la Diferencia Mínima Significativa (DMS, $\mathrm{P} \leq 0.05)$.

En 2009 y 2010 'Negro Comapa' se evaluó en un ensayo nacional de rendimiento (ENR), en el que se incluyeron cinco líneas avanzadas (CIAT-103-21, NGO 17-99, ELS15-55, NGO 007020 y NGO 007022) y cuatro variedades comerciales ('Negro Papaloapan', 'Negro 8025', 'Negro Guanajuato' y 'Negro Olinalá, del Programa de Frijol del INIFAP), junto con la variedad comercial 'Negro Michigan', material introducido de Estados Unidos y de amplio uso en el sureste de México.

El ENR se estableció en 13 ambientes de prueba en México: seis en Veracruz, cuatro en Guanajuato, uno en Durango, uno en Sinaloa y uno en Chiapas. En el Cuadro 1 se muestran las localidades, los años y la condición de humedad en que se condujo el ensayo, y las principales características ambientales de los sitios de prueba. El diseño experimental utilizado fue bloques completos al azar con cuatro repeticiones, con parcelas de tres surcos de $5 \mathrm{~m}$ de longitud, con separación de $0.60 \mathrm{~m}$. La parcela útil fue el surco central de cada parcela, al que se le eliminó $0.5 \mathrm{~m}$ de cada cabecera. Se hizo un análisis combinado del rendimiento de los 13 experimentos y se aplicó la prueba de separación de medias basada en la DMS $(\mathrm{P} \leq 0.05)$. También se hizo un análisis de regresión para comparar la adaptación de los genotipos con base en los coeficientes de determinación $\left(R^{2}\right)$ y regresión (bi) (Montgomery, 1991).

\section{Reacción a enfermedades}

Durante el periodo de conducción del ENR, en algunas localidades hubo incidencia natural de las enfermedades: virus del mosaico necrótico común del frijol (BCMNV) en Celaya, Gto., bajo riego; roya y tizón de halo en Celaya, Gto., bajo temporal; bacteriosis en Durango, Dgo., bajo riego; virus del mosaico común (BCMV) y mancha angular en Orizaba, Ver., bajo temporal; y virus del mosaico amarillo dorado del frijol (BGYMV) en Ocozocoautla, Chis., con humedad residual). La reacción de los genotipos a estas enfermedades se evaluó mediante el sistema 
Cuadro 1. Ciclos agrícolas, condición de humedad y características ambientales de los sitios de prueba de la variedad 'Negro Comapa' en México.

\begin{tabular}{|c|c|c|c|c|c|c|}
\hline Localidad/Estado & Año & Ciclo & Condición & Altitud(m) & $\begin{array}{l}\text { Temperatura } \\
\text { media }\left({ }^{\circ} \mathrm{C}\right)\end{array}$ & $\begin{array}{l}\text { Precipitación } \\
\text { pluvial (mm) }\end{array}$ \\
\hline Celaya, Gto. & 2009 & $\mathrm{I}-\mathrm{P}$ & Riego $^{\dagger}$ & 1754 & 20.6 & 597 \\
\hline Celaya, Gto. & 2009 & I-P & Riego $^{\dagger \dagger}$ & 1754 & 20.6 & 597 \\
\hline Durango, Dgo. & 2009 & $\mathrm{~V}$ & Riego & 1889 & 17.5 & 441 \\
\hline Celaya, Gto. & 2009 & V & Temporal ${ }^{\curvearrowleft}$ & 1754 & 20.6 & 597 \\
\hline Celaya, Gto. & 2009 & V & Temporal" & 754 & 20.6 & 597 \\
\hline Orizaba, Ver & 2009 & V & Temporal & 1248 & 19.0 & 2035 \\
\hline Orizaba, Ver. & 2010 & $\mathrm{~V}$ & Temporal & 1248 & 19.0 & 2035 \\
\hline Mazatlán, Sin. & $2009 / 2010$ & O-I & Riego & 3 & 24.1 & 800 \\
\hline Mtnez. de la Torre, Ver. & $2009 / 2010$ & O-I & $\mathrm{HR}$ & 151 & 24.0 & 1743 \\
\hline Medellín, Ver. & $2009 / 2010$ & O-I & $\mathrm{HR}$ & 15 & 25.4 & 1337 \\
\hline San Andrés Tuxtla, Ver. & $2009 / 2010$ & O-I & $\mathrm{HR}$ & 84 & 23.8 & 1750 \\
\hline Juan Rguez. Clara, Ver. & $2009 / 2010$ & O-I & $\mathrm{HR}$ & 148 & 26.3 & 1435 \\
\hline Ocozocoautla, Chis. & $2009 / 2010$ & O-I & $\mathrm{HR}$ & 864 & 23.6 & 898 \\
\hline
\end{tabular}

Fuentes: García (1987); Díaz et al. (2006); Serrano-Altamirano et al. (2006). ${ }^{\dagger}$ Ensayo sembrado el 20 de febrero; ${ }^{\dagger}$ Ensayo sembrado el 26 de febrero;

${ }^{\natural}$ Ensayo sembrado el 16 de julio; ${ }^{\Uparrow ⿻}$ Ensayo sembrado el 5 de agosto; I-P = ciclo invierno-primavera; $\mathrm{V}$ = ciclo de verano; O-I = ciclo otoño-invierno; $\mathrm{HR}=$ humedad residual.

estándar propuesto por CIAT (1987), que utiliza la escala de 1 a 9: 1 a $3=$ resistente, 4 a $6=$ intermedio, y 7 a $9=$ susceptible. Se calcularon correlaciones simples entre los valores promedio de la reacción de los genotipos a cada enfermedad con el rendimiento, para determinar si las enfermedades afectaron significativamente al rendimiento (Little y Hills, 1998).

\section{Evaluación de 'Negro Comapa' en parcelas de validación}

De 2009 a 2010 se establecieron 12 parcelas de validación en terrenos de agricultores cooperantes (10 en el Estado de Veracruz, una en Oaxaca y una en Chiapas), en las que 'Negro Comapa' se comparó con las variedades comerciales 'Negro Michigan' en condiciones de humedad residual y de temporal, y con 'Negro Jamapa' en condiciones de riego. La superficie por parcela varió de 2000 a $3000 \mathrm{~m}^{2}$, de acuerdo con la disponibilidad de semilla. El manejo agronómico del cultivo se hizo con base a las recomendaciones del INIFAP emitidas en sus respectivos Campos Experimentales (Villar, 1990; López et al., 1994). En cada parcela se hicieron cuatro muestreos al azar de un surco de $5 \mathrm{~m}$ de longitud y separación entre surcos de 0.60 $\mathrm{m}$, para comparar el rendimiento de grano de 'Negro Comapa' contra el de su correspondiente testigo, mediante la prueba t de Student (Reyes, 1990).

\section{RESULTADOS Y DISCUSIÓN}

\section{Rendimiento de 'Negro Comapa'}

En el ensayo regional (ERR), la línea CIAT-103-21 ('Negro Comapa') fue el genotipo de mayor rendimiento a través de los ambientes de evaluación, con un rendimiento promedio estadísticamente igual al de la línea ELS-11-37 y superior al del resto de los genotipos (Cuadro 2). En condiciones de temporal, 'Negro Comapa' superó en 131.6 y 45.9 $\%$ al rendimiento de los testigos Criollo regional y 'Negro Jamapa', respectivamente, mientras que en condiciones de humedad residual las superó en 20.2 y $15.5 \%$, respectivamente. Lo anterior confirmó la superioridad de esta nueva variedad en las diferentes áreas en las que se produce frijol en el Estado de Veracruz (López et al., 2011).

En el análisis combinado del ensayo nacional de rendimiento (ENR), se detectaron diferencias significativas $(\mathrm{P}<$ 0.05 ) entre ambientes (Cuadro 3 ), debidas principalmente a diferencias en las condiciones de humedad en que se condujeron los ensayos individuales. Los mayores rendimientos se obtuvieron en Durango, Dgo., Mazatlán, Sin. y Celaya, Gto, en condiciones de riego, los cuales superaron ( $\mathrm{P}<$ 0.05 ) a los obtenidos en el resto de las localidades. Los menores rendimientos se obtuvieron en Ocozocoautla, Chis. y en Juan Rodríguez Clara, Ver., sitios en los que el cultivo se desarrolló en condiciones de humedad residual, así como en Orizaba, Ver., bajo temporal; en la primera localidad el bajo rendimiento se debió principalmente a la incidencia del virus del mosaico amarillo dorado del frijol (BGYMV) 
Cuadro 2. Rendimiento promedio de 16 genotipos de frijol evaluados en el ensayo regional de rendimiento, en seis ambientes del Estado de Veracruz. Ciclos verano 2007, otoñoinvierno 2007/2008 y otoño-invierno 2009/2010.

\begin{tabular}{|c|c|c|}
\hline Genotipo & Origen & Rendimiento $\left(\mathrm{kg} \mathrm{ha}^{-1}\right)^{\dagger}$ \\
\hline CIAT-103-21 ('Negro Comapa') & CIAT & $1287.5 \mathrm{a}$ \\
\hline ELS-11-37 & INIFAP & $1184.8 \mathrm{ab}$ \\
\hline CIAT-103-25 & CIAT & $1140.2 \mathrm{bc}$ \\
\hline NGO-8-36 & INIFAP & $1119.3 \mathrm{bcd}$ \\
\hline NGO-17-99 & INIFAP & 1080.4 bcde \\
\hline CIAT-103-3 & CIAT & 1066.8 bcde \\
\hline CIAT-103-20 & CIAT & $1040.3 \mathrm{cde}$ \\
\hline ELS-15-55 & INIFAP & $1011.3 \mathrm{def}$ \\
\hline NGO-99-176 & INIFAP & 1003.9 def \\
\hline DOR-448 & CIAT & $994.5 \mathrm{def}$ \\
\hline 'Negro Jamapa' (Testigo comercial) & INIFAP & $991.7 \mathrm{def}$ \\
\hline $82 \mathrm{~L}-17-80$ & INIFAP & $988.6 \mathrm{ef}$ \\
\hline ELS-9-27 & INIFAP & $986.3 \mathrm{ef}$ \\
\hline ELS-14-48 & INIFAP & 984.7 ef \\
\hline DOR-454 & CIAT & $911.8 \mathrm{fg}$ \\
\hline Criollo regional (Testigo regional) & Dominio público & $798.5 \mathrm{~g}$ \\
\hline Promedio & & 1036.9 \\
\hline CV $(\%)$ & & 18.9 \\
\hline $\operatorname{DMS}(0.05)^{\dagger}$ & & 128.0 \\
\hline
\end{tabular}

Medias con letras iguales no son estadísticamente diferentes (DMS, 0.05). CV = coeficiente de variación; DMS (0.05) $=$ diferencia mínima significativa $(\mathrm{P} \leq 0.05)$.

Cuadro 3. Rendimiento promedio de grano de frijol en 13 ambientes del ensayo nacional de rendimiento de frijol negro opaco. 2009 y 2010.

\begin{tabular}{lccccc}
\hline Localidad/Estado & Año & Ciclo & Condición & CV(\%) & Rendimiento $\left(\mathrm{kg} \mathrm{ha}^{-1}\right)$ \\
\hline Durango, Dgo. & 2009 & $\mathrm{~V}$ & Riego & 13.14 & $3145.1 \mathrm{a}$ \\
Mazatlán, Sin. & $2009 / 2010$ & O-I & Riego & 9.53 & $2999.0 \mathrm{a}$ \\
Celaya, Gto. & 2009 & I-P & Riego $^{\dagger}$ & 18.08 & $2915.2 \mathrm{a}$ \\
Celaya, Gto. & 2009 & I-P & Riego $^{\dagger \dagger}$ & 15.31 & $2618.9 \mathrm{~b}$ \\
San Andrés Tuxtla, Ver. & $2009 / 2010$ & O-I & Humedad residual $^{+}$ & 11.15 & $2255.6 \mathrm{c}$ \\
Orizaba, Ver. & 2009 & V & Temporal & 16.26 & $1608.0 \mathrm{~d}$ \\
Celaya, Gto. & 2009 & V & Temporal" & 24.64 & $1360.8 \mathrm{e}$ \\
Celaya, Gto. & 2009 & V & Temporal & 33.95 & $1033.3 \mathrm{f}$ \\
Mtnez. de la Torre, Ver. & $2009 / 2010$ & O-I & Humedad residual & 21.94 & $973.5 \mathrm{f}$ \\
Medellín, Ver. & $2009 / 2010$ & O-I & Humedad residual & 22.13 & $657.2 \mathrm{~g}$ \\
Orizaba, Ver. & 2010 & V & Temporal & 29.01 & $504.0 \mathrm{gh}$ \\
Juan Rguez. Clara, Ver. & $2009 / 2010$ & O-I & Humedad residual & 21.89 & $441.0 \mathrm{gh}$ \\
Ocozocoautla, Chis. & $2009 / 2010$ & O-I & Humedad residual & 27.97 & $353.3 \mathrm{~h}$ \\
DMS (0.05) & & & & 238.5 \\
\hline
\end{tabular}

${ }^{\dagger}$ Ensayo sembrado el 20 de febrero; ${ }^{\dagger \dagger}$ Ensayo sembrado el 26 de febrero; "Ensayo sembrado el 16 de julio; ${ }^{\uparrow \oplus E n s a y o ~ s e m b r a d o ~ e l ~} 5$ de agosto; Medias con letras iguales no son estadísticamente diferentes (DMS, 0.05). CV = coeficiente de variación; DMS $(0.05)=$ diferencia mínima significativa $(\mathrm{P} \leq 0.05)$. 
que afectó significativamente la mayoría de los genotipos en evaluación (Villar et al., 2003); en la segunda localidad se debió a deficiencias de humedad en las etapas de formación de vainas R7 y de llenado de vainas R8, y en la tercera, a la presencia del patógeno causante de la mancha angular (Phaoisariopsis griseola) a partir de la etapa de prefloración R5 hasta la etapa R8 (Fernández et al., 1986; López et al., 2010).

También se detectaron diferencias significativas entre genotipos $(\mathrm{P}<0.01)$. En el Cuadro 4 se muestra que las variedades 'Negro Comapa' y 'Negro Papaloapan' fueron las de mayor rendimiento dentro del grupo sobresaliente; estos dos genotipos superaron en rendimiento al testigo 'Negro Michigan' y a la variedad 'Negro Olinalá, que fueron las de menor rendimiento.

De acuerdo con los parámetros de estabilidad propuestos por Eberhart y Russell (1966), todos los genotipos fueron estables en su comportamiento, ya que sus coeficientes de regresión y desviaciones de regresión: fueron $b i=1$ y $S^{2} d i$ $=0$. Los altos valores de $R^{2}$ registrados en la respuesta de la línea NGO 007022 y en la de las variedades 'Negro Papaloapan' y 'Negro Comapa', indican mayor predictibilidad en su respuesta a los ambientes, mientras que los genotipos con los menores valores corresponden a los de bajo rendimiento en sitios con presión de enfermedades y de deficiencia de humedad, o de alta respuesta en el rendimiento en alguna localidad específica (López et al., 2002b) como el caso de 'Negro Michigan' y la variedad 'Negro Olinalá' que produjeron los menores rendimientos en presencia del virus de mosaico común (BCMV), mancha angular y virus del mosaico amarillo dorado del frijol (BGYMV), así como de sequía, en tanto que en condiciones de riego estos mismos genotipos registraron altos rendimientos (datos no mostrados).

\section{Reacción a enfermedades}

De las enfermedades que ocurrieron en forma natural en el ENR, sólo el virus del mosaico común (BCMV) y la mancha angular en Orizaba, Ver. bajo condiciones de temporal, y el virus del mosaico amarillo dorado del frijol (BGYMV), en Ocozocoautla, Chis. con humedad residual, afectaron significativamente $(\mathrm{P}<0.05)$ el rendimiento de los genotipos de frijol. 'Negro Comapa' mostró resistencia a las dos primeras enfermedades y fue tolerante al BGYMV, mientras que 'Negro Michigan' fue susceptible a las tres enfermedades (Cuadro 5). Estos resultados indican que con la siembra de la variedad 'Negro Comapa', los productores pueden disminuir riesgos de daño por enfermedades, sobre todo porque la mayoría de ellos no aplica agroquímicos para su control (López et al., 2002a).

\section{Comportamiento de 'Negro Comapa' en parcelas de validación}

Los rendimientos obtenidos en las parcelas de validación indicaron que esta nueva variedad fue superior a 'Negro Jamapa' y 'Negro Michigan' en las tres condiciones de humedad estudiadas (temporal, humedad residual y riego). El rendimiento promedio general de 'Negro Comapa' fue 51 $\%$ mayor que el de los testigos regionales (Cuadro 6). La validación de genotipos permitió verificar los resultados experimentales en cuanto a productividad y reacción a factores adversos a nivel semi-comercial, bajo las condiciones

Cuadro 4. Rendimiento promedio y parámetros de estabilidad de 10 genotipos de frijol evaluados en 13 ambientes de México. 2009 y 2010.

\begin{tabular}{lccccc}
\hline Genotipo & Origen & Rendimiento $\left(\mathrm{kg} \mathrm{ha}^{-1}\right)$ & $b i$ & $S^{2} d i$ & $R^{2}$ \\
\hline 'Negro Comapa' & CECOT & $1764.5 \mathrm{a}$ & 1.06 & 9303.16 & 0.9327 \\
'Negro Papaloapan' & CECOT & $1713.6 \mathrm{a}$ & 0.96 & -26068.94 & 0.9462 \\
'Negro 8025' & CEVAMEX & $1689.3 \mathrm{ab}$ & 1.12 & -35958.60 & 0.4384 \\
NGO 17-99 & CECOT & $1664.0 \mathrm{ab}$ & 0.94 & -23865.93 & 0.2915 \\
ELS 15-55 & CECOT & $1663.7 \mathrm{ab}$ & 0.92 & -69059.54 & 0.3591 \\
'Negro Guanajuato' & CEBAJ & $1617.0 \mathrm{ab}$ & 1.05 & -76404.74 & 0.4054 \\
NGO 007020 & CEBAJ & $1599.1 \mathrm{ab}$ & 0.93 & -44880.18 & 0.4075 \\
NGO 007022 & CEBAJ & $1575.1 \mathrm{ab}$ & 1.10 & -46934.92 & 0.9716 \\
'Negro Michigan' & USA & $1483.0 \mathrm{bc}$ & 1.09 & 14638.14 & 0.3449 \\
'Negro Olinalá' & CEBAJ & $1280.6 \mathrm{c}$ & 0.84 & 18759.43 & 0.2287 \\
Promedio & & 1605.0 & & & \\
DMS (0.05) & & 209.2 & & & \\
\hline
\end{tabular}

CECOT $=$ Campo Experimental Cotaxtla-INIFAP; CEVAMEX = Campo Experimental Valle de México-INIFAP; CEBAJ = Campo Experimental Bajío-INIFAP; Medias con letras iguales no son estadísticamente diferentes (DMS, 0.05$) ; b i=$ coeficiente de regresión; $S^{2} d i=$ desviación de regresión; $R^{2}=$ coeficiente de determinación. 
de suelo, clima y manejo de los agricultores (Ugalde et al., 2005). 'Negro Comapa' mostró mayor potencial de rendimiento que los materiales de mayor uso comercial. Además, su grano reúne las características físicas y de calidad que demandan los consumidores del sureste de México (López et al., 2011).

\section{CONCLUSIONES}

La variedad 'Negro Comapa' mostró alto rendimiento (de 1287 a $1764 \mathrm{~kg} \mathrm{ha}^{-1}$ ) y amplia adaptación en las áreas tropicales y en el altiplano de México, así como tolerancia al virus del mosaico amarillo dorado del frijol, y resistencia al virus del mosaico común y a la mancha angular. Esta variedad fue liberada y registrada en el Catálogo Nacional de Variedades Vegetales con el número FRI-066-100910, y su siembra comercial es recomendada principalmente para los estados del sureste de México.
En diversas localidades, 'Negro Comapa' superó en rendimiento a los genotipos mejorados experimentales, y en siembras de agricultores superó a variedades comerciales.

El rendimiento obtenido por 'Negro Comapa' a través de las localidades de prueba fue dependiente en primera instancia de la disponibilidad de humedad durante el ciclo del cultivo, y modificado en algunas localidades por la presencia de patógenos causantes de enfermedades foliares.

\section{AGRADECIMIENTOS}

Al Fondo Sectorial Secretaría de Agricultura, Ganadería, Pesca y Alimentación (SAGARPA) - Consejo Nacional de Ciencia y Tecnología (CONACYT), por el apoyo proporcionado al proyecto S0007 2009-1 109621, a través del cual se financiaron las actividades de investigación y validación de 2009 a 2011.

Cuadro 5. Reacción a enfermedades de la variedad 'Negro Comapa' y tres testigos comerciales evaluados en diferentes localidades de México. 2009-2010.

\begin{tabular}{|c|c|c|c|}
\hline Genotipo & $\mathrm{BCMV}^{\dagger}$ & Mancha angular $^{\dagger \dagger}$ & BGYMV ${ }^{\star}$ \\
\hline 'Negro Comapa' & 2.00 & 2.50 & 5.25 \\
\hline 'Negro Guanajuato' & 3.75 & 4.75 & 6.75 \\
\hline 'Negro 8025’' & 3.25 & 5.00 & 5.50 \\
\hline 'Negro Michigan' & 3.75 & 8.00 & 6.50 \\
\hline Correlación RG vs. ENF & $r=-0.683^{\star}$ & $\mathrm{r}=-0.883^{\star \star}$ & $r=-0.687^{\star}$ \\
\hline
\end{tabular}

Cuadro 6. Rendimiento ( $\left.\mathrm{kg} \mathrm{ha}^{-1}\right)$ de la variedad 'Negro Comapa' y de testigos comerciales en parcelas de validación establecidas en los Estados de Veracruz Oaxaca y Chiapas.

\begin{tabular}{lcccccc}
\hline Localidad/Estado & Año & Ciclo & Condición & 'Negro Comapa' & Testigos & $\begin{array}{c}\text { Incremento respecto } \\
\text { al testigo (\%) }\end{array}$ \\
\hline Medellín, Ver. & $2008 / 2009$ & O-I & HR & 1292 & 983 & 31.4 \\
Orizaba, Ver. & $2008 / 2009$ & O-I & HR & 1462 & 1026 & 42.5 \\
José Azueta, Ver. & 2009 & I-P & HR & 777 & 822 & -5.5 \\
Isla, Ver. & $2009 / 2010$ & O-I & HR & 2000 & 639 & 213.0 \\
Tuxtepec, Oax. & $2009 / 2010$ & O-I & HR & 1202 & 1032 & 16.5 \\
San Andrés Tuxtla, Ver. & $2009 / 2010$ & O-I & HR & 2340 & 1399 & 67.3 \\
Juan Rguez. Clara, Ver. & 2010 & I-P & HR & 429 & 115 & 273.0 \\
José Azueta, Ver. & 2010 & I-P & HR & 764 & 636 & 20.1 \\
Ocozocoautla, Chis. & $2010 / 2011$ & O-I & HR & 1720 & 630 & 173.0 \\
José Azueta, Ver. & 2011 & I-P & HR & 1294 & 644 & 100.9 \\
Promedio & & & & 1328.0 & 792.6 & 67.5 \\
Orizaba, Ver. & V 2009 & & Temporal & 1917 & 1750 & 9.5 \\
Medellín, Ver. & I-P 2010 & & Riego & 1622 & 1450 & 11.9 \\
Promedio total & & & & $1401.6^{* *}$ & 927.2 & 51.2 \\
\hline
\end{tabular}

${ }^{\dagger}$ En condiciones de riego se utilizó como testigo a la variedad 'Negro Jamapa' y en humedad residual y temporal a la variedad 'Negro Michigan' y 'Criollo Sesentano' en Ocozocoautla, Chis.; O-I = otoño-invierno. I-P = invierno-primavera. $\mathrm{V}=$ verano. $\mathrm{HR}=$ humedad residual. ${ }^{*}$ Diferencia significativa $(\mathrm{t}$ Student, $\mathrm{P}<0.01)$. 


\section{BIBLIOGRAFÍA}

Acosta G J A, F M Mendoza H, B Aguilar G, G Esquivel E, R Rodríguez G, S H Guzmán M (2008) Negro Guanajuato, nueva variedad de frijol para el centro de México. Agric. Téc. Méx. 34:107-111.

Acosta G J A, R Rosales S, R Navarrete M, E López S (2000) Desarrollo de variedades mejoradas de frijol para condiciones de riego y temporal en México. Agric. Téc. Méx. 26:79-98.

Castellanos Z J, H. Guzmán-Maldonado, A Jiménez, C Mejía, J de J Muñoz-Ramos, J A Acosta-Gallegos, G Hoyos, E López-Salinas, D González-Eguiarte, R Salinas-Pérez, J González-Acuña, J A Muñoz-Villalobos, P Fernández-Hernández, B Cáceres (1997) Hábitos preferenciales de los consumidores de frijol común (Phaseolus vulgaris L.) en México. Arch. Latinoam. Nutr. 47:163-167.

CIAT, Centro Internacional de Agricultura Tropical (1987) Sistema Estándar para la Evaluación de Germoplasma de Frijol. A van Schoonhoven, A M Pastor-Corrales (comps). CIAT, Cali, Colombia. $56 \mathrm{p}$.

Díaz P G, J A Ruiz C, M A Cano G, V Serrano A, G Medina G (2006) Estadísticas Climatológicas Básicas del Estado de Veracruz (Período 1961-2003). Libro Técnico No. 13. SAGARPA. INIFAP. CIRGOC. Campo Experimental Cotaxtla. Veracruz, México. $292 \mathrm{p}$.

Eberhart S A, W A Russell (1966) Stability parametrs for comparing varieties. Crop Sci. 6:36-40

Fernández F, P Gepts, M López (1986) Etapas de desarrollo de la planta de frijol común (Phaseolus vulgaris L.). CIAT, Cali, Colombia. $34 \mathrm{p}$.

García E (1987) Modificaciones al Sistema de Clasificación Climática de Köppen (para adaptarlo a las condiciones de la República Mexicana). 4a ed. Universidad Nacional Autónoma de México. México, D. F. 130 p.

Little M T, F J Hills (1998) Métodos Estadísticos para la Investigación en la Agricultura. 2da ed. P Crespo (trad). Ed. Trillas. México, D. F. $270 \mathrm{p}$.

López S E, A Durán P, E N Becerra L, V A Esqueda E, O Cano R (1994) Manual de Producción de Frijol en el Estado de Veracruz. Folleto para Productores No. 7. SARH. INIFAP. CIRGOC. Campo Experimental Cotaxtla. Cotaxtla, Veracruz, México. 29 p.

López S E, J A Acosta G, J Cumpián G, O Cano R, B Villar S, E N Becerra L (2002b) Adaptación de genotipos de frijol común en la región tropical húmeda de México. Agric. Téc. Méx. 28:35-42.

López S E, J A Acosta G, O Cano R, G Fraire V, E N Becerra L, B Villar S, J Cumpián G, F J Ugalde A (2002a) Negro Tropical, Nueva Variedad de Frijol para el Trópico Húmedo de México. Folleto Técnico No. 32. SAGARPA. INIFAP. CIRGOC. Campo Experimental Cotaxtla. Veracruz, México. 13 p.

López S E, J A Acosta G, O H Tosquy V, R A Salinas P, B M Sánchez G, R Rosales S, C González R, T Moreno G, B Villar S, H M Cortinas E, R Zandate H (2011) Estabilidad de rendimiento en genotipos mesoamericanos de frijol de grano negro en México. Rev. Mex. Cien. Agríc. 2:29-40.
López S E, O H Tosquy V, B Villar S, E N Becerra L, F J Ugalde A, J Cumpián G (2006) Adaptabilidad de genotipos de frijol resistentes a enfermedades y a suelos ácidos. Rev. Fitotec. Mex. 29:33-39.

López S E, O H Tosquy V, B Villar S, J R Rodríguez R, F J Ugalde A, A Morales R, J A Acosta G (2010) Negro Comapa, nueva variedad de frijol para el estado de Veracruz. Rev. Mex. Cien. Agríc. 1:715-721.

López S E, O H Tosquy V, B Villar S, J R Rodríguez R, F J Ugalde A, A Morales R, J A Acosta G (2011) Negro Comapa, Nueva Variedad de Frijol para el Estado de Veracruz. Folleto Técnico No. 55. SAGARPA. INIFAP. CIRGOC. Campo Experimental Cotaxtla. Veracruz, México. 24 p.

Montgomery D C (1991) Diseño y Análisis de Experimentos. Versión en español. Grupo Editorial Iberoamérica, S. A. de C. V. México, D. F. 589 p.

Olivares S E (1994) Paquete Estadístico de Diseños Experimentales (Programa de cómputo) versión 2.5. Facultad de Agronomía de la Universidad Autónoma de Nuevo León. Marín, Nuevo León, México.

Reyes C P (1990) Diseño de Experimentos Aplicados Agronomía, Biología, Química, Industrias, Ciencias Sociales y Ciencias de la Salud. 3a ed. Ed. Trillas. México, D. F. 348 p.

SAGARPA, Secretaría de Agricultura, Ganaderia, Desarrollo Rural, Pesca y Alimentación (2011) Anuarios Estadísticos de la Producción Agrícola. Servicio de Información Agroalimentaria y Pesquera (SIAP). SAGARPA. México, D. F. Disponible en: http://www.siap.sagarpa.gob.mx [Julio 2011].

Serrano-Altamirano V, G Díaz-Padilla, A López-Luna, M A Cano-García, A D Báez-González, E R Garrido-R (2006) Estadísticas Climatológicas Básicas de Chiapas (Período 1961-2003). Libro Técnico No. 1. SAGARPA. INIFAP. CIRPAS. Campo Experimental Centro de Chiapas. Ocozocoautla de Espinosa, Chiapas, México. 186 p.

Servicio de Información y Estadística Agroalimentaria y Pesquera, SIAP (2006) Situación Actual y Perspectivas de la Producción de Frijol en México 2000-2005. Servicio de Información y Estadística Agroalimentaria y Pesquera. Secretaría de Agricultura, Ganadería, Desarrollo Rural, Pesca y Alimentación (SAGARPA). México. 34 p.

Singh S P, H F Schwartz (2010) Breeding common bean for resistance to diseases: a review. Crop Sci. 50:2199-2223.

Singh S P, P Gepts, D G Debouck (1991) Races of common bean (Phaseolus vulgaris, Fabaceae). Econ. Bot. 45:379-396.

Ugalde A F J, E López S, O H Tosquy V (2005) Validación de genotipos de frijol en la zona central del estado de Veracruz, México. Agron. Mesoam. 16:1-5.

Villar S B (1990) Guía para Cultivar Frijol en Chiapas. Folleto para Productores No. 1. SARH. INIFAP. CIAPAS. Tuxtla Gutiérrez, Chiapas, México. $20 \mathrm{p}$.

Villar S B, E López S, J A Acosta G (2003) Selección de genotipos de frijol por rendimiento y resistencia al mosaico dorado y suelos ácidos. Rev. Fitotec. Mex. 26:109-114.

Villar S B, E López S, O H Tosquy V (2009) Negro Grijalva, nuevo cultivar de frijol para el trópico húmedo de México. Agric. Téc. Méx. 35:349-352. 